\title{
Software Vampp Bovino como instrumento de mediación dialógica entre el sector productivo bovino y la academia
}

\author{
Vampp Bovino Software As Instrument for the Dialogic \\ Mediation Between Academia and the Bovine Productive \\ Sector
}

\author{
Juan José Romero Zúñiga \\ Universidad Nacional \\ Heredia, Costa Rica \\ juan.romero.zuniga@una.cr \\ José Rojas Campos \\ Universidad Nacional \\ Heredia, Costa Rica \\ jrojas@una.cr \\ Miguel Bolaños Segura \\ Universidad Nacional \\ Heredia, Costa Rica \\ miguel.bolanos.segura@una.cr \\ Gloriana Castillo Badilla \\ Universidad Nacional \\ Heredia, Costa Rica \\ gloriana.castillo.badilla@una.cr \\ Bernardo Vargas Leitón \\ Universidad Nacional \\ Heredia, Costa Rica \\ bernardo.vargas.leiton@una.cr \\ Sandra Estrada König \\ Universidad Nacional \\ Heredia, Costa Rica \\ sandra.estrada.konig@una.cr
}

Recibido: 10/05/2019 Aceptado: 30/08/2019 
Revista Universidad EN DiÁLOGo • Vol. 9, N. ํ2, Julio-Diciembre, 2019 • 99-116

ISSN 2215-2849 • EISSN: 2215-4752

URL: http://www.revistas.una.ac.cr/index.php/dialogo/index Correo electrónico: universidadendialogo@una.cr DOI: http://doi.org/10.15359/udre.9-2.5

Resumen. El programa informático VAMPP Bovino es un paquete de apoyo a la toma de decisiones en fincas de ganado bovino, bubalino y porcino, originalmente desarrollado en la Universidad de Utrecht (Países Bajos), pero traído a Costa Rica, en 1986, por medio de un programa de cooperación internacional (MHO de los Países Bajos) a la Escuela de Medicina Veterinaria de la UNA. Aquí, en el Centro Regional de Informática para la Producción Animal Sostenible (CRIPAS), el programa se adaptó y validó a las condiciones productivas costarricenses, en primera instancia, para luego trascender fronteras y ubicarse en toda Centroamérica, México, el Caribe y varios países de Suramérica. Este proceso ha sido posible, en primer lugar, por el respaldo de la UNA a este proyecto que, de hecho, es el único en su tipo, en todo el mundo, que sobrevive una vez que el financiamiento MHO cesó; pero sin duda por el proceso de comunicación y diálogo constante con los actores de los distintos segmentos del sector productivo ganadero de Costa Rica -y de los otros países en donde se usa VAMPP-. Este proceso dialógico que ha recogido, y sigue recogiendo, el conocimiento y las experiencias de quienes realizan o son responsables de toda la cadena de producción, transformación y comercialización de los productos bovinos es el que hace posible su incorporación en un programa informático que ha beneficiado a más de dos mil productores en diversos países, especialmente más de 1500 en Costa Rica, así como a todos los participantes de la cadena de producción, pero con especial efecto en el consumidor que tiene productos de buena calidad (alimento seguro) y en cantidad (seguridad alimentaria). VAMPP Bovino es un modelo de proyecto universitario exitoso, llevado bajo el respaldo de la UNA.

Palabras clave: universidad, diálogo de saberes, investigación acción participativa, informática, desarrollo.

Abstract. The VAMPP Bovino software is a package to support decision-making in cattle, buffalo and pig farms. It was originally developed at the University of Utrecht (Netherlands) and brought to Costa Rica in 1986, as part of an international cooperation project (MHO program, Netherlands), to the School of Veterinary Medicine of the National University of Costa Rica (UNA). Here, at the Regional Center of Informatics for Sustainable Animal Production (CRIPAS), in the first instance, the program was adapted and validated to the Costa Rican productive conditions, to later transcend borders and be implemented throughout Central America, Mexico, the Caribbean and several countries of South America. This process has been possible, first of all, thanks to the support of the UNA to this project which, in fact, is the only one of its kind in the world that survives once the MHO financing ceased. Undoubtedly, this also has been possible thanks to the process of communication and constant dialogue with the stakeholders of the different segments of the livestock production sector of Costa Rica and the other countries where VAMPP is used. This dialogical process, which has gathered -and continues gathering- the knowledge and experiences of the people 
URL: http://www.revistas.una.ac.cr/index.php/dialogo/index

CORREO ELECTRÓNICO: universidadendialogo@una.cr

DOI: http://doi.org/10.15359/udre.9-2.5

who are involved in, or are responsible for, the entire chain of bovine products, from production until consumption, is what makes it possible to incorporate them into a computer program that has benefited more than 2000 producers in different countries, especially more than 1500 in Costa Rica. Besides, it benefited all the participants in the production chain, but with special effect on the consumer who has good quality products (safe food) in good quantity (food safety). VAMPP Bovino is a successful university project model, carried out under the support of UNA.

Keywords: university, knowledge dialog, participatory action research, informatics, development.

\section{Introducción}

\section{Antecedentes sobre VAMPP}

La Escuela de Medicina Veterinaria (EMV) de la Universidad Nacional (UNA) nace casi con la UNA misma allá por 1974 (http://www.medvet.una.ac.cr/index. php/acercade/resena-historica). Desde ese mismo momento, la EMV tenía como propósito fundamental servir como fuente de profesionales en medicina veterinaria con una alta vocación hacia las especies productivas, con el objetivo de fortalecer los sistemas de producción existentes $\mathrm{y}$, a mediano plazo, apoyar el nacimiento y la mejora de sistemas productivos poco tradicionales.

En 1986, como parte de ese proceso de revisión de contenidos programáticos, y dadas las necesidades de incrementar la eficiencia de los sistemas productivos bovinos, especialmente de lechería, se inicia un proyecto de intercambio entre la EMV-UNA y la Universidad de Utrecht (Países Bajos), denominado Proyecto Salud de Hato, con el objeto de incluir en el currículum de la EMVUNA el enfoque de Salud de Hato y Control de la Producción (PSHCP), que había sido desarrollado con enorme éxito en aquel país europeo.

Estos PSHCP incluyen metodologías integradas y herramientas sistemáticas y sistematizadas que permiten el manejo apropiado de datos para la generación de información, con el objetivo de apoyar la toma oportuna de decisiones adecuadas para el sistema mismo. Es así como, en 1986, llega a Costa Rica la versión 2.1 de VAMPP (siglas por su nombre en inglés: Veterinary Automated Management and Production control Programme: Noordhizen \& Buurman, 1984), un programa de cómputo desarrollado en la Universidad de Utrecht (Países Bajos) que permitía monitorear diferentes aspectos de los PSHCP en lechería bovina (Pérez et al., 1989). 
Revista Universidad en Diálogo • Vol. 9, N. ${ }^{\circ}$ 2, Julio-Diciembre, 2019 • 99-116

ISSN 2215-2849 • EISSN: 2215-4752

URL: http://www.revistas.una.ac.cr/index.php/dialogo/index CoRreo ElECtrónico: universidadendialogo@una.cr DOI: http://doi.org/10.15359/udre.9-2.5

En este esfuerzo por la introducción de la metodología de los PSHCP se logra suscribir un importante proyecto de cooperación entre el Gobierno de los Países Bajos y el de Costa Rica, por medio de la Universidad de Utrecht (UU) y la UNA, respectivamente. Como parte de este proyecto se realizó un fuerte intercambio de académicos y estudiantes de los Países Bajos y de Costa Rica, especialmente para la introducción, adopción y adaptación e implementación de los PSHCP y, principalmente, del software VAMPP (Capella et al., 1991).

Una vez en la EMV-UNA, el VAMPP es primero traducido al español, para luego ser adaptado y validado en dos proyectos piloto en dos cuencas lecheras: Poás y Tilarán. Cada proyecto incluyó cerca de veinticinco fincas en cada uno, de distintos tamaños, intensidades de producción y tipo de productores, siendo de lechería especializada en Poás, y de doble propósito en Tilarán.

En 1990, se ofrece al mercado nacional la primera versión adaptada y validada para las condiciones de Costa Rica, denominada VAMPP Leche 3.0. Luego vinieron las versiones VAMPP Leche 3.1, 3.2, 4.0 y 5.0; finalmente, se liberó, en 1995, la versión VAMPP Leche 5.1.

Durante el período entre 1995 y 2002, se desarrolló la versión VAMPP Bovino 1.0, que vendría a tomar el lugar de VAMPP Leche 5.1, con el fin de cubrir la necesidad de un programa que incluyera los distintos sistemas de producción bovina e integrase la mayoría de los requerimientos fundamentales de las fincas lecheras: doble propósito, carne y cría. Así, en el año 2007, sale al mercado la versión VAMPP Bovino 2.0 y, en 2010, se libera al mercado la versión VAMPP Bovino 3.0. (Romero et al., 2011a), la cual, inclusive, permite el monitoreo y control de hatos de búfalos de agua.

Desde ese momento hasta la fecha, los cambios han sido de tal magnitud que los mismos creadores del programa original han reconocido que las versiones mejoradas en Costa Rica constituyen, prácticamente, un nuevo programa. Lo más relevante en esta evolución es que todo ha sido producto del proceso constante de realimentación mediada por el diálogo entre los participantes activos del sistema. De ese modo, el acercamiento fue gestado primero desde la EMV-UNA hacia los productores que, con el tiempo, hizo que esos mismos productores, fuera en forma individual o gremial, buscaran a la academia en busca de respuestas a sus problemas, en un proceso de constante diálogo, que aún se mantiene y es, más bien, más patente y fuerte. 


\section{¿Qué es CRIPAS?}

A finales de los años noventa, en este proceso dinámico de mejora académica de la EMV-UNA, siempre buscando ofrecer los mejores profesionales en medicina veterinaria a la sociedad costarricense y centroamericana -pues muchos centroamericanos se formaban en esta escuela-, así como dar a los sistemas productivos resultados de investigación pertinente y de calidad, se crea el Centro Regional de Informática para la Producción Animal Sostenible (CRIPAS), que vino a consolidar el proceso catalítico en ese diálogo entre los productores y la academia, siempre teniendo a VAMPP como su elemento comunicante (Romero et al., 2011a). Posteriormente, por aspectos administrativos de la UNA, se cambia la palabra Centro por Consultoría dentro del nombre de CRIPAS, por lo que actualmente es la Consultoría Regional de Informática para la Producción Animal Sostenible.

Casi veinte años después, CRIPAS se ha consolidado en el imaginario de los productores y sus organizaciones gremiales, siendo un actor fundamental en el desarrollo de investigación pertinente para la continua mejora del VAMPP, que, en este momento, ofrece su versión VAMPP Bovino 3.0. Esta versión cuenta con una amplia gama de utilidades que le permiten el más completo monitoreo del sistema de producción al ganadero, tanto en lechería especializada como en ganadería de cría y engorde, en apoyo a la toma de decisiones oportunas sobre una base técnicamente fundamentada, así como es un apoyo crucial para los asesores técnicos de los sistemas, tales como médicos veterinarios, zootecnistas, ingenieros agrónomos, economistas agrícolas, entre otros (Romero et al., 2011a).

Adicionalmente, y de una forma no menos importante, VAMPP Bovino y CRIPAS son fuente constante de consulta por parte de organizaciones gubernamentales, no gubernamentales y privadas, tales como el Servicio Nacional de Salud Animal (SENASA), de la Cámara Nacional de Productores de Leche y de las principales empresas agroindustriales productoras y transformadoras de leche y carne.

CRIPAS es un programa integrado de la UNA, con muchos años de actividad permanente en la transferencia de tecnología informática pecuaria que se desarrolla en la EMV-UNA en estrecha relación con el Programa de 
Revista Universidad en Diálogo • Vol. 9, N. ${ }^{\circ}$ 2, Julio-Diciembre, 2019 • 99-116

ISSN 2215-2849 • EISSN: 2215-4752

URL: http://www.revistas.una.ac.cr/index.php/dialogo/index CorReo ElECtrónico: universidadendialogo@una.cr DOI: http://doi.org/10.15359/udre.9-2.5

Investigación en Medicina Poblacional (MedPob) y del Posgrado Regional en Ciencias Veterinarias Tropicales (PCVET), así como con distintas cátedras de la misma EMV. Presta servicios al sector productivo, tanto a productores individuales como a asociaciones de productores, asesores, centros de capacitación, centros o instituciones de investigación, extensión y docencia y organizaciones e instituciones oficiales y privadas ligadas a la producción animal en América Latina (Romero et al., 2011a; VAMPP Bovino 3.0, 2018).

Este programa tiene como eje central de su labor el desarrollo y la validación de sistemas de información y métodos de análisis, así como la investigación de las necesidades de los propietarios, responsables y asesores de los sistemas de producción nacionales e internacionales, que hacen uso de sus programas de cómputo VAMPP Bovino 3.0 y VAMPP Porcino; siendo su producto estrella el software Bovino. En este sentido, la investigación está orientada a mejorar las características técnicas que VAMPP ofrece a sus usuarios para lograr sistemas de producción más eficientes, con el óptimo uso de los recursos que disponen, reduciendo la dependencia de insumos y obteniendo el óptimo económico de las fincas de una forma sostenida y en armonía con el ambiente (VAMPP Bovino 3.0, 2018).

Actualmente, cuenta con la versión Bovino 3.0 que funciona en casi dos mil fincas, tanto en Costa Rica como en otros países de América Latina y el Caribe, desde México hasta Bolivia, de las cuales casi 1500 son costarricenses, lo que ha permitido que CRIPAS sea el centro de referencia en la búsqueda de datos e información para el sector lechero especializado para los distintos actores de la cadena de producción láctea costarricense (Romero et al., 2011a; Romero et al., 2011b; Carmona, 2013; León, 2017).

\section{Rol de los sistemas de información automatizados en los PSHCP}

Una máxima de la era de la tecnología de la información y la comunicación dice que quien tenga la información adecuada en el momento oportuno tendrá la posibilidad de tomar las mejores decisiones. En el caso de los sistemas de producción animal, resulta casi imposible pensar en que un productor que tiene en su finca un agronegocio tome las decisiones basado en corazonadas, intuiciones o en otras razones que no sean la información fidedigna que el mismo sistema de producción genera. En este sentido, los Sistemas de Información para el Manejo de la Finca (SIMF) se convierten en herramientas esenciales en el apoyo a la toma de decisiones, en primera instancia en la finca misma, pero buscando ir más allá, permitiendo analizar los datos de varios sistemas de producción, de forma que se logre crear información general 
URL: http://www.revistas.una.ac.cr/index.php/dialogo/index

CORREO ELECTRÓNICO: universidadendialogo@una.cr

DOI: http://doi.org/10.15359/udre.9-2.5

sobre la realidad del sector, desde la particularidad de todos y cada uno de los sistemas de producción (Baaijen \& Pérez, 1995).

No solo se debe pretender que el sistema sea eficaz porque produce leche, carne o animales para la venta; más allá de eso, y de forma imprescindible, hay que detallar cuán eficiente es el sistema, esto determinado mediante indicadores zootécnicos o bioeconómicos. Asimismo, es crucial que todo sistema de producción apunte a la sostenibilidad (económica, social y ambiental), buscando producir alimentos inocuos en suficiente cantidad y calidad. En este entramado de condiciones, el sistema de información se reafirma como el elemento esencial para controlar y monitorear todas las actividades del sistema de producción y determinar si las metas y objetivos de la finca se cumplen o no (figura 1).
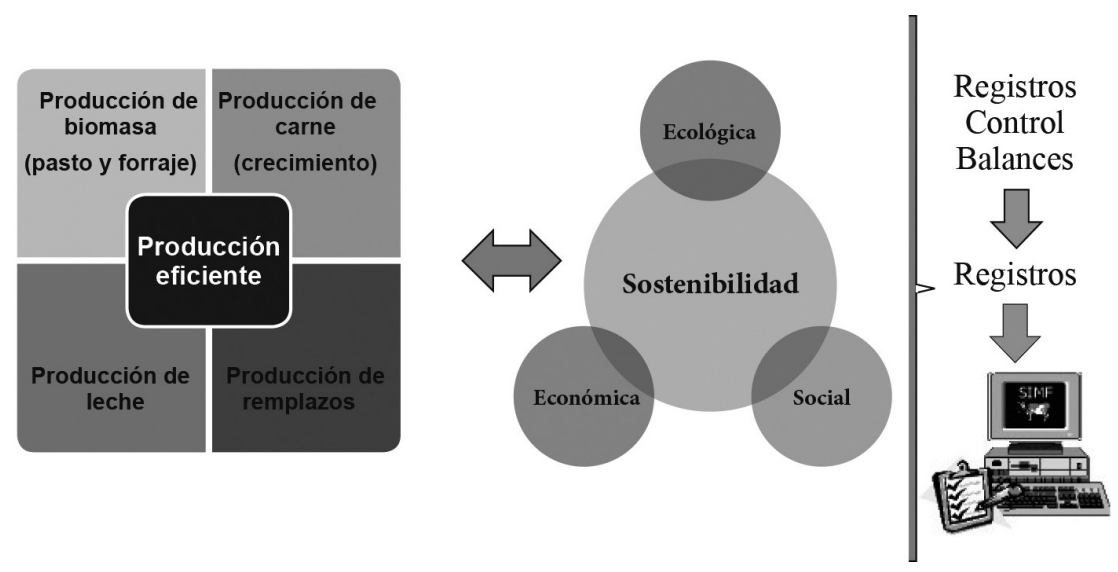

Figura 1. Relación producción-sostenibilidad y el rol que desempeñan los SIMF.

Fuente: Romero et al., 2011.

Los datos, en la finca, cumplen con un ciclo que va desde su generación y captura en las distintas áreas de trabajo (crianza de terneras, reproducción, producción de leche o carne, nutrición y alimentación, salud, personal, finanzas, etc.) hasta que se convierten en información procesada y disponible para la toma de decisiones por parte del dueño de la finca o del administrador, asesorado muchas veces por un profesional. Pero para esto, los datos deben haber sido capturados e introducidos en el SIMF mediante un proceso riguroso, constante y confiable. Así, los datos individuales e independientes producidos por cada unidad de producción o los actores del sistema de producción se convierten en información útil para la toma oportuna de decisiones adecuadas (figura 2). 


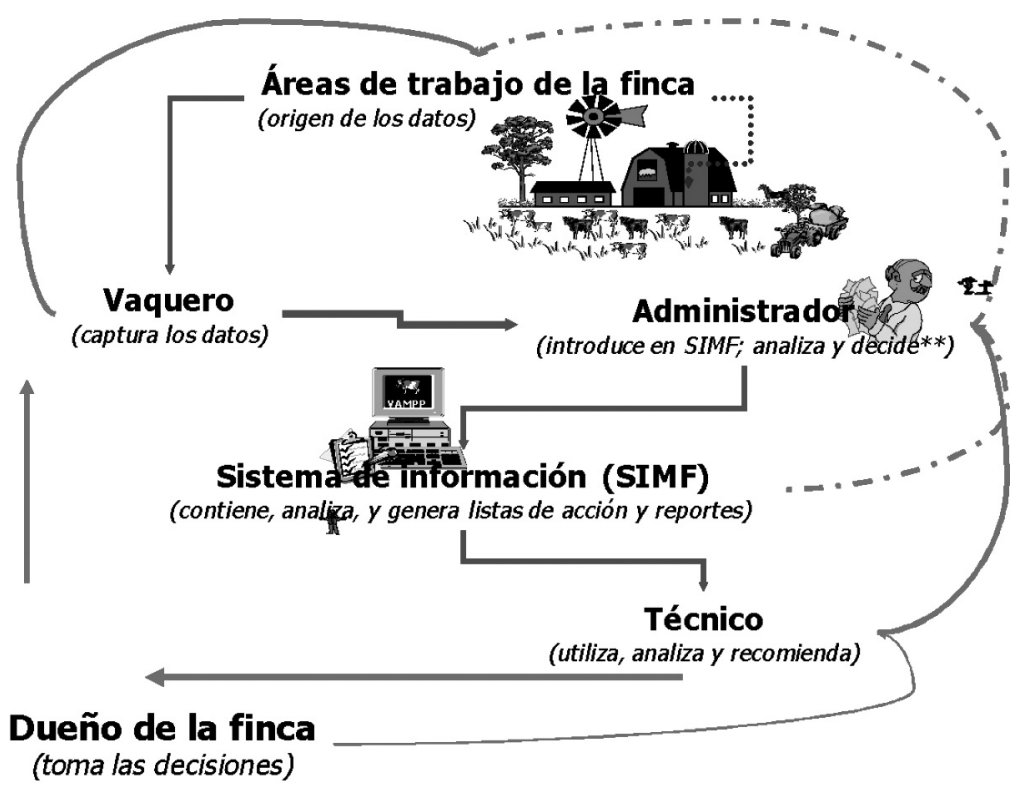

Figura 2. Ciclo de los datos e información dentro del sistema de producción.

Fuente: Romero et al., 2011.

\section{Proceso dialógico comunidad productiva-academia y beneficios conexos}

Desde sus inicios, tanto el Proyecto de Salud de Hato como su sucesor CRIPAS fueron y siguen siendo practicantes, quizás hasta de una forma un tanto intuitiva, de la Investigación Acción Participativa (IAP) (Eizagirre y Zabala, s.f; Colmenares, 2012). En esta metodología de trabajo, los actores de la cadena de producción bovina, o sea, los grupos o colectivos originalmente pensados como objeto a investigar han jugado un rol protagónico en el proceso del desarrollo del software, pues se han constituido, más bien, en sujetos de la investigación (Alberich, 2008). De ese modo, se ha seguido la ruta de pensamiento de Fals (1981), que dice que la IAP:

Se relaciona más con una actividad de investigación propia de la base popular sobre su realidad, que con una acción receptiva de investigaciones realizadas por élites de intelectuales ajenas a ellas. En la InvestigaciónAcción Participativa, el científico social se enfrenta a la necesidad de compartir los objetivos y los métodos mismos con la base social. Ya no es investigación para" las masas, sino que surge de la base social misma". 
Hay que dejar claro que, si bien en CRIPAS no hay investigadores sociales propiamente dichos, su forma de trabajo ha ido allende la metodología tradicional de investigación verticalista en la que quien conoce es quien está en la academia, sino que reconoce que en el proceso creador del conocimiento propio y autóctono quienes mejor conocen son los mismos actores sociales.

Desde ese punto de vista, a pesar de que en un principio el acercamiento fue desde la academia hacia los productores de la forma tradicional -tomándolos como objeto de estudio-, con el tiempo hubo un cambio en el paradigma y el enfoque de trabajo, pues, de una manera espontánea, los mismos productores comprendieron su rol preponderante en la producción del conocimiento sobre la base de sus experiencias acumuladas por décadas, así como por la vivencia diaria.

Esto es, en esencia, un proceso de IAP (Fals, 1999; Alberich, 2008). De igual modo, otros participantes en la cadena productiva, llegando hasta el mismo consumidor, han dado su aporte a la investigación y desarrollo del VAMPP, pues con el reforzamiento de la inocuidad de alimentos y de la filosofía de "de la finca al plato" los sistemas de producción animal han tenido que adaptarse a los cambios constantes, con tal de mantenerse vigentes y, en ese proceso, mediante el constante diálogo de saberes entre los responsables de la cadena de producción -en todos sus niveles- y los académicos del Proyecto Salud de Hato, y posteriormente con CRIPAS, han surgido las mejoras al software VAMPP hasta llevarlo a ser una herramienta de amplia utilidad para la toma de decisiones, desde lo individual (sobre la vaca) hasta lo gremial (cámaras de productores) o la agroindustria (Romero et al., 2011), según la información que los mismos sistemas generan, pero basado en las necesidades que los actores han definido como tales.

Este aprendizaje mutuo que permite el diálogo de saberes, producto mismo de la IAP, entre los actores de toda la cadena de producción y consumo de los productos de origen bovino y los académicos de la EMV-UNA, ha sido posible por la acción catalizadora que el software VAMPP ha representado para todos los sectores en las formas que a continuación se describen.

\section{Sector primario de producción y sector académico}

El proceso dialógico se ha dado porque los académicos no han sido impositivos en su concepción de lo que los sistemas de producción deben ser desde lo que la ciencia dice y establece, sino que ha trabajado en conjunto con los productores atendiendo sus consultas, abriendo espacio a sus inquietudes, escuchando sus 
Revista Universidad en Diálogo • Vol. 9, N. ${ }^{\circ}$ 2, Julio-Diciembre, 2019 • 99-116

ISSN 2215-2849 • EISSN: 2215-4752

URL: http://www.revistas.una.ac.cr/index.php/dialogo/index Correo electrónico: universidadendialogo@una.cr DOI: http://doi.org/10.15359/udre.9-2.5

sugerencias e, inclusive, tomando como elementos de investigación prácticas culturales históricas para darles sustento científico, de modo que fueran reproducibles por otros productores en otros entornos.

Esta máxima cobra especial importancia pues, aunque sea duro de aceptar, la mayoría del conocimiento sobre sistemas de producción animal, específicamente el bovino, ha sido una importación de aquel generado en otras latitudes, muchas veces en condiciones ambientales, culturales y de manejo que no se acercan a las costarricenses: es otro ecosistema que requiere mucha investigación para llegar a un verdadero enfoque ecosistémico (Maltby, 2000; Berbés et al., 2014) para las condiciones propias de Costa Rica o de los otros países en que se ha adoptado a VAMPP como sistema de información para la toma de decisiones.

En ese proceso, las propuestas desde la academia han sido evaluadas en los mismos sistemas de producción con la participación activa de los productores, quienes, de manera desinteresada, han sometido a prueba las hipótesis de investigación en sus propias fincas y animales, teniendo como herramienta de monitoreo el VAMPP, para luego analizar los datos utilizando métodos estadísticos apropiados y robustos. Al final, si el diseño del estudio fue el apropiado, son los mismos productores quienes se encargan de difundir el conocimiento logrado (González, 2012).

Asimismo, en otras ocasiones han sido más bien los mismos productores los que se han acercado a la academia con una serie de propuestas (problemas), en busca de respuestas, pero con la certeza de formar parte del proceso de investigación y de incorporación del conocimiento nuevo generado. De nuevo, el diálogo de saberes se ha impuesto de una forma espontánea de aplicación de la IAP. Al final, el software VAMPP se ve fortalecido porque, además de servir de herramienta para el monitoreo durante la investigación, con alguna frecuencia se le hacen inclusiones de nuevos módulos de captura de datos y análisis de información, así como de indicadores productivos más apropiados para los sistemas de producción costarricenses (Campus, 2007).

Todo esto ha redundado en un sistema de información nacional que, a partir de los datos de cada productor, se ha consolidado en la gran Base de Datos VAMPP, ubicada en CRIPAS (Romero et al., 2011), posibilitando que un productor se beneficie a sí mismo y a sus colegas, pues con esta nube de información se obtienen indicadores más objetivos y ajustados a la realidad de la producción nacional, lo que sin duda ofrece mejor sustento para la toma de decisiones (Carmona, 2013; Sánchez, 2018). 
URL: http://www.revistas.una.ac.cr/index.php/dialogo/index

CORREO ELECTRÓNICO: universidadendialogo@una.cr

DOI: http://doi.org/10.15359/udre.9-2.5

\section{Sector transformador y sector académico}

Desde un inicio, debido a la forma de organización del sector lechero costarricense, en el que una cooperativa, específicamente la Cooperativa de Productores de Leche R.L. (Dos Pinos), agremia a cerca de 1550 productores de todo el país, que a su vez transforma el producto primario para darle valor agregado y ofrecerlo al mercado nacional e internacional en forma de muy diversos productos, se hizo posible que el diálogo entre productorestransformadores y la academia se diera, de nuevo, en forma natural y espontánea.

Para lograr mejores productos para el consumidor, los transformadores requieren el mejor producto primario posible, en este caso, leche de la mejor calidad nutricional e higiénica. En ese proceso de búsqueda del mejor y mayor producto obtenido en el propio inicio del eslabón de la cadena: la vaca. Desde la academia se han conducido numerosas investigaciones con el objetivo de establecer las condiciones en que se puede conseguir que las vacas produzcan la mayor cantidad de leche, de la mejor calidad, inocua, de manera sostenible y teniendo el bienestar animal como elemento transversal en el proceso (Romero et al., 2000; Romero et al., 2011c; González, 2012).

Unido a esto, está la presión por hacer más eficiente todo el sistema de producción-transformación-comercialización, sin tener grandes fluctuaciones en el producto de origen que causen problemas en el abastecimiento del producto terminado que se ofrece al consumidor final. Esto requiere que el sector transformador pueda, con la mayor certeza posible, predecir los niveles de producción primaria y sus calidades, con alguna anticipación para establecer los contratos comerciales respectivos. En ese aspecto, VAMPP Bovino ha permitido a la Dos Pinos hacer proyecciones -a varios meses plazo- sobre la cantidad y la calidad de la leche que recibirá y que podrá transformar, con niveles de precisión que rondan el 97\% (León, 2012).

En este proceso dialógico, buscando que el sector de producción primaria logre la colocación de todo su producto, que el sector transformador cuente con el insumo de la mayor calidad higiénica y nutricional sin poner en riesgo el abastecimiento en el mercado, y que el consumidor tenga a su disposición productos lácteos de primera calidad y en suficiente cantidad a precios competitivos, la IAP ha cumplido un rol fundamental buscando la máxima eficiencia en todo el proceso. Al final, el mismo VAMPP Bovino se ha visto fortalecido en su estructura, pues se le han incorporado módulos de monitoreo de los elementos productivos que hacen posible un proceso con alta eficiencia a pesar del elevado grado de complejidad. 


\section{Sector industrial y sector académico}

Otro sector con el que CRIPAS ha establecido un diálogo constante es el de la industria de bienes utilizados en la producción bovina; de ese modo, farmacéuticas, fabricantes de alimentos concentrados y suplementos alimenticios, distribuidores de balanzas electrónicas, representantes y distribuidores de salas de ordeño, entre otros, se han acercado a CRIPAS para, atendiendo necesidades de los mismos productores, incorporar soluciones informáticas en el VAMPP, de modo que muchos de los procesos de control de inventarios de medicamentos, alimentos concentrados o suplementos alimenticios, costeo de la producción, control de pesos y medidas, rendimiento en matadero, etc., que son cruciales para incrementar la eficiencia en el sistema de producción, ofreciendo el máximo aprovechamiento para el productor y el mínimo de incertidumbre en el industrial, han sido incorporados en VAMPP para su debido monitoreo.

Varias empresas que distribuyen insumos pecuarios en fincas bovinas han incursionado en el uso de VAMPP Bovino para monitorear los efectos que surten los tratamientos que ellas mismas recomiendan, de modo que el productor puede ver, de manera objetiva, los resultados de esas intervenciones, casi a tiempo real. Esta forma de trabajo ha ganado adeptos en forma rápida, de modo que varias empresas usan ya el VAMPP como fuente de datos e información para dar una consultoría más adecuada a sus clientes.

A modo de ejemplo, la cantidad de alimento concentrado que se consume en una finca depende, principalmente, del inventario animal, del estado reproductivo, de la edad y del nivel de producción. Al industrial le interesa conocer con la mayor certidumbre posible esos elementos proyectados al futuro en términos de meses y VAMPP le provee esa información, de modo que puede administrar la logística de importación de los insumos y de producción, haciendo un uso eficiente de instalaciones, equipo, recurso humano y finanzas (León, 2012).

De ese modo, la eficiencia en el proceso industrial redundará en un beneficio directo al productor que no pagará por las ineficiencias del industrial y, a su vez, esa ganancia en el proceso se trasladará al consumidor porque los costos de producción serán más apropiados. En todo este proceso, VAMPP ofrece las herramientas instrumentales de tipo informático que permiten mayor eficiencia en la cadena productiva.

Todos estos usos, monitoreados en el VAMPP, permiten el análisis de las políticas y formas de trabajo del sector industrial, de modo que, de forma 
URL: http://www.revistas.una.ac.cr/index.php/dialogo/index

CORREO ELECTRÓNICO: universidadendialogo@una.cr

DOI: http://doi.org/10.15359/udre.9-2.5

objetiva y con datos fiables, VAMPP ha ofrecido datos en alta cantidad con inmejorable calidad, para estudios orientados hacia la economía agrícola y de la administración de agronegocios.

\section{Sector oficial y sector académico}

Un actor preponderante, por su rol de rector, es el Servicio Nacional de Salud Animal (SENASA), con quien también CRIPAS ha establecido procesos de diálogo constante, siempre buscando las mejores soluciones desde la óptica y las necesidades del sector productor, pero bajo la regencia del SENASA. En muchas ocasiones CRIPAS ha servido de fuente de datos e información de primera mano para que el sector oficial planifique o tome decisiones, así como para la participación en investigaciones en que VAMMPP es fuente de información.

Un ejemplo palpable de los productos tangibles surgidos de ese proceso dialógico entre el sector oficial y el académico a través de CRIPAS es que, con el advenimiento de la Ley de Control de Ganado Bovino, Prevención y Sanción de su Robo, Hurto y Receptación ( $\mathrm{N}^{\mathrm{o}} 8799$ ), se hace necesaria la implementación de un sistema único de identificación de fincas y de animales que permita el control de la movilización de los semovientes a lo interno del país, o para su exportación.

Como parte de ese proceso, el SENASA creó el Sistema Integrado de Registro de Establecimientos Agropecuarios (SIREA) que, además, contiene un módulo de Trazabilidad Individual. Según este sistema, cada animal de cada finca debe contar con su identificación única que, unida a la identificación única de la finca, produce una identidad irrepetible de cada animal. En este caso, en VAMPP se han incorporado los elementos que permitan esa identificación única; además, en conjunto con algunas empresas distribuidoras de equipos de identificación individual automatizados, se logró incorporar en VAMPP una serie de opciones que permiten el registro automatizado de la identificación de cada animal y que, en caso de robo o hurto, o simple movilización por venta, permitan una identificación segura y certera.

\section{Sector académico}

CRIPAS es un programa más de los varios existentes en la EMV-UNA que, gracias a la robusta e invaluable base de datos VAMPP, se mantiene en constante diálogo con otros proyectos y programas de la misma EMV, así como con el sector docente en general, pues provee de datos e información 
Revista Universidad en Diálogo • Vol. 9, N. ${ }^{\circ}$ 2, Julio-Diciembre, 2019 • 99-116

ISSN 2215-2849 • EISSN: 2215-4752

URL: http://www.revistas.una.ac.cr/index.php/dialogo/index CoRreo ElECtrónico: universidadendialogo@una.cr DOI: http://doi.org/10.15359/udre.9-2.5

que dan sustento a otros proyectos de investigación, extensión y docencia. Es así como se ha aportado datos a unas ocho tesis doctorales, cerca de un par de docenas de tesis de maestría y no menos cantidad de tesis de licenciatura. En ese proceso de interacción con el resto de los académicos de la EMV han surgido necesidades por datos o indicadores particulares que, al final de cada proyecto, se han ido incorporando al VAMPP. Además, producto de los proyectos de investigación, en cada uno de ellos, siempre se hace una validación de los datos, de modo que el control de la calidad de los datos y la creación de módulos y rutinas que garanticen la fiabilidad de los datos desde su captura hasta su análisis y presentación ha sido una ganancia neta de VAMPP por la participación activa de la academia misma en la investigación.

Esta relación academia-academia, en que ambas partes aportan en un proceso participativo continuo por medio de la investigación aplicada ha permitido, además, que el nuevo conocimiento generado sea incorporado en el corpus de contenidos de los cursos que se ofrecen a los estudiantes de la carrera de Medicina Veterinaria durante su formación. Pero el beneficio no se queda solo en la EMV, sino que ha llegado a otras escuelas de la misma UNA (Ciencias Agrarias y Ciencias Ambientales) y a otras universidades del ámbito nacional, como la Universidad de Costa Rica, la Universidad Técnica Nacional y el Instituto Tecnológico, así como también en el ámbito internacional, a la Escuela Agrícola Panamericana El Zamorano (Honduras) y la Facultad de Medicina Veterinaria y Zootecnia de la Universidad Nacional Autónoma (UNAM) de México, entre las más importantes (Garelli, 2018).

De ese modo, los estudiantes reciben conocimiento actualizado, pero más importante aún, producido en forma autóctona, de los ecosistemas productivos en los que se desenvolverá como profesional, con conocimiento producido en consonancia con una realidad nacional y producto del constante diálogo de saberes de todos quienes participan de forma activa, a todo lo largo de la cadena productiva, comercial y de consumo del sistema bovino $\mathrm{y}$, muy importante también, del consumidor final.

De modo general, es posible afirmar que VAMPP ha sido y sigue siendo un catalizador del conocimiento generado en los mismos sistemas de producción, en las más diversas condiciones climáticas y de manejo, con la participación de los productores y en diálogo con la academia mediante la IAP, incorporando al resto de los actores de la cadena de producción (figura 3 ). 


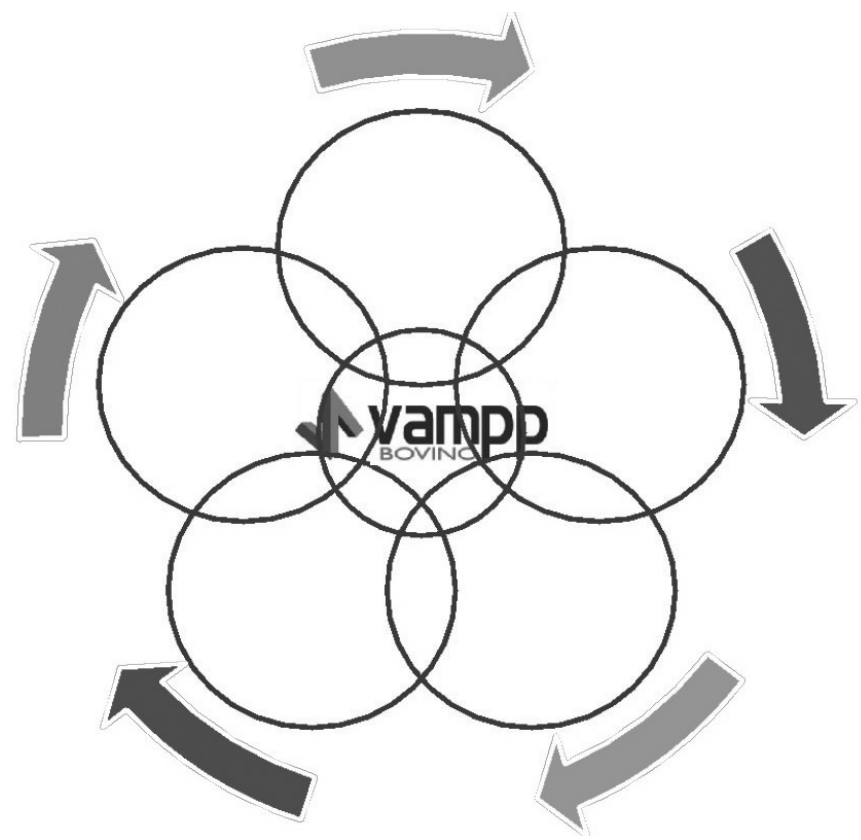

Figura 3. VAMPP Bovino como eje de conexión de los actores de la cadena productiva bovina.

Fuente: Elaboración propia.

\section{Conclusiones}

Los procesos de creación del conocimiento, producto del intercambio de información entre distintas entidades con la consecuente aprehensión e incorporación de ella en sus corpus de saberes, se ven reforzados cuando un proceso dialógico con alta asertividad se instaura entre ellas; con más razón cuando este mecanismo está mediado por la academia. Es menester de los académicos prestar atención a lo que la sociedad les comunica, aún muchas veces en forma tácita; es más, debe estar preparado para identificar las más imperceptibles señales en la comunidad para acercarse a ella e iniciar, de forma respetuosa y responsable, el diálogo que impulsará la creación del conocimiento mediante el intercambio o la gestación de nuevos saberes. 
En este papel, primero el Proyecto de Salud de Hato, y luego CRIPAS, utilizando como medio catalizador al software VAMPP, han logrado importantes productos que dan fe de los saberes generados en conjunto con los distritos actores del sector productivo ganadero costarricense, desde y hacia ellos. Este es, desde nuestra perspectiva, un claro ejemplo de que proyectos integrados academia-sociedad pueden lograr importantes frutos, no importa si se trata de iniciativas público-público o público-privado.

\section{Referencias bibliográficas}

Alberich, T. (2008). IAP, redes y mapas sociales: desde la investigación a la intervención social. Portularia, 8(1), 131-151.

Baaijen, M. \& Pérez, E. (1995). Information technology in the Costa Rican dairy sector: A key instrument in extension and on-farm research. Agric Hum Values, 12(2), 45-51. doi: https://doi.org/10.1007/BF02217296

Berbés, M., Oestreicher, J. S., Mertens, F. y Saint-Charles, J. (2014). Ecohealth and resilience thinking: a dialog from experiences in research and practice. Ecology and Society, 19(2), 24. http://dx.doi.org/10.5751/ ES-06264-190224

Campus (2007). Nueva versión de VAMPP Bovino: un software para la ganadería. Recuperado de http://www.campus.una.ac.cr/ ediciones/2007/julio/2007julio_pag05.html

Cappella, E., Buurman, J., Pérez, E., Baaijen, M. T. y Muller, E. (1991). Development and uptake of a livestock information system in Costa Rica, part 2: uptake and validation of VAMPP software in Costa Rican dairy farms. In Proceedings XXIV World Veterinary Congress. Rio de Janeiro, Brazil.

Carmona, G. (2013). Los retos de hoy en la reproducción en ganado de leche: factores clave que nos afectan y cómo enfrentarlos. Memorias, XIX Congreso Lechero Nacional. 4-5 diciembre 2013. Heredia, Costa Rica.

Colmenares, A. M. (2012). Investigación-acción participativa: una metodología integradora del conocimiento y la acción. Voces y Silencios: Revista Latinoamericana de Educación, 3(1), 102-115. doi: https://doi. org/10.18175/vys3.1.2012.07 
Eizagirre, M. y Zabala, N. (s. f.). Investigación-acción participativa. Diccionario de acción humanitaria y cooperación al desarrollo. Recuperado de http://www.dicc.hegoa.ehu.es/listar/mostrar/132

Fals, O. (1981). La ciencia y el pueblo en investigación participativa y praxis rural. Lima: Moxa Azul Editores.

Fals, O. (1999). Orígenes universales y retos actuales de la IAP (investigación acción participativa). Análisis Político, 38(2), 71-88.

Garelli, O. (2018). Análisis de la eficacia de la cooperación México-Costa Rica. Caso de estudio: texto didáctico multimedia de reproducción de bovinos dirigido a productores. Oxfam México.

González, L. M. (2012). Programa UNA Mirada, Canal 13. Publicado el 6 de agosto, 2012. Recuperado de https://www.youtube.com/ watch? $\mathrm{v}=$ PefK7nNC1pU\&index=133\&list=PLD5F19CA7FA896144

León, H. (2012). Programa UNA Mirada, Canal 13. Publicado el 6 de agosto, 2012. Recuperado de https://www.youtube.com/ watch? $v=$ PefK7nNC1pU\&index=133\&list=PLD5F19CA7FA896144

León, H. (2017). La economía de la reproducción. XXIII Congreso Nacional Lechero, Costa Rica.

Maltby, E. (2000). Ecosystem approach: from principle to practice. Ecosystem Service and Sustainable Watershed Management in North China International Conference, Beijing, P.R.

Noordhuizen, J. P. \& Buurman, J. (1984). VAMPP: a veterinary automated management and production control programme for dairy farms (the application of MUMPS for data processing). Vet Q. 6(2), 66-72. doi: https://doi.org/10.1080/01652176.1984.9693914

Pérez, E., Baiijen, M., Capella, E. \& Barkema, H. (1989). Development of a livestock information system for Costa Rica. Livestock Production and diseases in the Tropics. Proceedings of the VI International Conference for Tropical Veterinary Medicine, Utrecht, The Netherlands.

Romero, J. J., Estrada, S. y Pérez, E. (11-15 setiembre, 2000). Uso del sistema de información VAMPP en investigaciones a gran escala. XVII Congreso Panamericano de Ciencias Veterinarias. 
Romero, J. J., Rojas, J. A. y Estrada, S. (2011a). El programa VAMPP Bovino como herramienta de apoyo a la toma de decisiones en los sistemas de producción bovinos. Ventana Lechera, 16, 4-14.

Romero, J. J., Rojas, J. A., Estrada, S. y Bolaños, M. (2011b). Análisis de datos (re)productivos utilizando la base nacional de datos VAMPP. Ventana Lechera, 16, 26-41.

Romero, J. J., Rojas, J. A., Estrada, S., Bolaños, M. y Vargas, B. (2011c). Análisis de datos (re)productivos utilizando muestra de investigaciones realizadas con el programa VAMPP Bovino como fuente de base de datos. Ventana Lechera, 16, 57-66.

Sánchez, G. Z. (2018). Análisis bioeconómico para correlacionar variables de eficiencia productiva, reproductiva y de salud con los niveles de adopción del programa VAMPP ${ }^{\circledR}$ en fincas lecheras y de doble propósito en Costa Rica. Tesis de maestría. Universidad Nacional Autónoma de Mexico, CD de México.

SENASA-MAG. Sistema de registro de establecimientos agropecuarios (SIREA). http://www.senasa.go.cr/sistemas-en-linea/VAMPP Bovino 3.0. 2018. Recuperado de http://vampp-cr.com/creditos.htm). Visitado: 11 noviembre de 2018 . 